\title{
Comparative Evaluation of Mechanical and Microleakage Properties of Cention-N, Composite, and Glass Ionomer Cement Restorative Materials
}

\author{
Ramachandra Sujith ${ }^{1}$, Tej G Yadav ${ }^{2}$, Deepti Pitalia ${ }^{3}$, Prashant Babaji ${ }^{4}$, Kommula Apoorva ${ }^{5}$, Ankita Sharma ${ }^{6}$
}

\begin{abstract}
Aim: The longevity of restorative materials depends on the resistance to masticatory forces. The present study was undertaken to evaluate the mechanical and microleakage properties of Cention- $\mathrm{N}$ with glass ionomer cement (GIC) and composite restorative materials.

Materials and methods: A total of 45 specimen blocks were prepared with 15 samples of each type of restorative material, namely Cention-N, GIC, and hybrid composite. Samples were subjected to load at crosshead speed of $0.75 \pm 0.25 \mathrm{~mm} / \mathrm{min}$ till the fracture of sample. Class V cavities were prepared on the buccal surface of orthodontically extracted premolars followed by restoration of each test material. All the surfaces of the tooth were coated with clear nail varnish except $1 \mathrm{~mm}$ around the margins of the restorations. These samples were immersed in $0.5 \%$ basic fuchsine dye and longitudinally sectioned and observed under stereomicroscope to check microleakage. The obtained data were statistically evaluated. Results: We found the highest mean compressive and flexural strength for hybrid composite followed by Cention-N and least for GIC which is statistically significant $(p<0.001)$.

Conclusion: Mean microleakage was least for Cention-N. Cention-N is a newer restorative material having higher mechanical properties with lesser microleakage.

Clinical significance: Cention- $\mathrm{N}$ is a newer restorative material having promising properties. This material can be used as an alternative restorative material.

Keywords: Cention-N, Glass ionomer cement, Hybrid composite, Properties.

The Journal of Contemporary Dental Practice (2020): 10.5005/jp-journals-10024-2837
\end{abstract}

\section{INTRODUCTION}

Dental caries is one of the most common causes for loss of tooth structure, which impairs shape and function of the affected tooth. Tooth damaged with dental caries can be restored with various restorative materials. A restorative material is one which reestablishes the esthetic, functional, and biological properties of the tooth structure. ${ }^{1}$ Various direct filling materials are available in dentistry such as amalgam, glass ionomer cement (GIC), and esthetic composite material. The longevity of the restorative materials depends on the resistance to masticatory forces (compressive and flexural) and lower microleakage. ${ }^{1,2}$ Each of these restorative materials has certain advantages and disadvantages; hence, proper selection of restorative materials will help for its clinical success. ${ }^{3}$

Marginal seal is an important factor for the success of restoration. Microleakage is the clinically undetectable passage of bacteria, fluid, molecules, or ions in microgaps $(10-6 \mu \mathrm{m})$ between the cavity wall and the restorative material applied to it. Lower the microleakage score of restorative material, longer will be the survival of restoration in oral cavity. ${ }^{3}$

Amalgam was first introduced to dentistry in the 19th century. It was widely used since ages, but due to its certain disadvantages such as higher coefficient of thermal expansion, unesthetic look, mercury toxicity, and microleakage, a substitute material was tried. Glass ionomer cement has a wide range of applications in dentistry. It chemically bonds to the tooth structure and has anticaries effect. But it has low abrasion resistance, high solubility, poor mechanical properties, and questionable compressive strength. ${ }^{3,4}$
${ }^{1}$ Department of Conservative Dentistry, Sharavathi Dental College, Shimoga, Karnataka, India

${ }^{2}$ Department of Pediatric and Preventive Dentistry, Goenka Research Institute of Dental Sciences, Gandhinagar, Gujarat, India

${ }^{3}$ Department of Conservative Dentistry and Endodontics, Index Institute of Dental Sciences, Indore, Madhya Pradesh, India

${ }^{4}$ Department of Pedodontics, Sharavathi Dental College, Shimoga, Karnataka, India

${ }^{5}$ Department of Conservative Dentistry and Endodontics, Malla Reddy Institute of Dental Sciences, Suraram, Hyderabad, Telangana, India

${ }^{6}$ Department of Conservative Dentistry and Endodontics, Maharshi Markandeshwar College of Dental Sciences and Research, Mullana, Ambala, Haryana, India

Corresponding Author: Prashant Babaji, Department of Pedodontics, Sharavathi Dental College, Shimoga, Karnataka, India, Phone: +91 8971096312, e-mail: babajipedo@gmail.com

How to cite this article: Sujith R, Yadav TG, Pitalia D, et al. Comparative Evaluation of Mechanical and Microleakage Properties of Cention-N, Composite, and Glass lonomer Cement Restorative Materials. J Contemp Dent Pract 2020;21(6):691-695.

Source of support: Nil

Conflict of interest: None

Composites are tooth-colored restorative materials widely used for anterior and posterior restoration due to excellent esthetic and minimal tooth reduction. But it is a time-consuming procedure. ${ }^{3}$

(c) The Author(s). 2020 Open Access This article is distributed under the terms of the Creative Commons Attribution 4.0 International License (https://creativecommons. org/licenses/by-nc/4.0/), which permits unrestricted use, distribution, and non-commercial reproduction in any medium, provided you give appropriate credit to the original author(s) and the source, provide a link to the Creative Commons license, and indicate if changes were made. The Creative Commons Public Domain Dedication waiver (http://creativecommons.org/publicdomain/zero/1.0/) applies to the data made available in this article, unless otherwise stated. 
Cention-N is a new tooth-colored esthetics material with high flexural strength. It belongs to group of Alkasites. It is a subgroup of composite material class. The $\mathrm{pH}$ value during acid attacks will be regulated by increased hydroxide ions release from alkaline filler. It has the properties of amalgam and $\mathrm{GIC}^{1,3-5}$

There are very few reported studies on mechanical and microleakage properties of Cention-N. Hence, the present study was undertaken to compare and evaluate the mechanical properties and microleakage of Cention-N with GIC and hybrid composite restorative materials.

\section{Materials and Methods}

This in vitro study was conducted in Index Institute of Dental Science, Indore, after obtaining approval from institutional ethical committee. The study was done to evaluate the mechanical (compressive and flexural strength) and microleakage properties of restorative materials.

\section{Flexural and Compressive Strength Evaluation}

A total of 45 specimen blocks were prepared with 15 samples of each type of restorative material, namely group I: Cention- $\mathrm{N}$ (Ivoclar-Vivadent, Mumbai, India), group II: GIC (GC Fuji), and group III: hybrid composite (3M Filtek Z250XT) (Figs 1 and 2). Samples of each restorative material were fabricated using metal split molds of $3 \pm 1 \mathrm{~mm}$ (diameter) $\times 5 \pm \mathrm{mm}$ (height) to evaluate compressive and flexural strength. These prepared samples were stored at $37 \pm 1^{\circ} \mathrm{C}$ for 24 hours in a water bath before testing. Samples were subjected to universal testing machine (Fig. 2) connected to load-measuring cell with load applied at crosshead speed of $0.75 \pm 0.25 \mathrm{~mm} / \mathrm{min}$ till the fracture of sample.

\section{Microleakage Evaluation}

Orthodontically extracted 45 premolars free from crack, caries, restoration, or white spot lesion on the buccal surface were selected. Teeth were cleaned and stored in distilled water until use. Class $V$ cavities on the buccal surface of premolars were prepared of dimension $2 \mathrm{~mm}$ depth, $3 \mathrm{~mm}$, width $2 \mathrm{~mm}$, and height in occlusogingival direction using high-speed flat end straight diamond bur (SF-41 ISO 109/010 Mani Dia Burs) with water coolant. All the tooth preparations were randomly divided into three groups of 15 samples each and restored with respective materials, that is group I (Cention-N), group II (GIC), and group III (hybrid composites) (Figs 3 to 5). All the procedures were done by a single trained investigator.

All restored teeth were stored in distilled water at $37^{\circ} \mathrm{C}$ for 24 hours. Later, they were subjected to 200 thermocycles at $5^{\circ} \mathrm{C}$ and $55^{\circ} \mathrm{C}$ lasted for 30 seconds to simulate the variation in oral thermal condition. The root apices were sealed with sticky wax (to prevent any dye leakage through the apex) followed by two-coat painting to all the crown and root surfaces of the tooth with clear nail varnish except $1 \mathrm{~mm}$ around the margins of the restorations (Figs 3 to 5). These samples were immersed in $0.5 \%$ basic fuchsine dye at $37^{\circ} \mathrm{C}$ for 24 hours, followed by washing in water and dried. Later, each tooth was longitudinally sectioned through the center of restoration with the help of diamond disk under water coolant. All sections were observed under stereomicroscope at $20 \times$ magnification. After observing each longitudinal section of a particular tooth, a section with greater microleakage was selected for scoring. The leakage distance from the margins to the determined limit was recorded in millimeter. Mean leakage score for all groups were calculated and evaluated.

Munro, Hilton, and Hermesch scoring system used for scoring the leakage in the present study. ${ }^{6}$

Score 0: No evidence of microleakage;

Score 1: Penetration of dye up to half of cavity depth;

Score 2: Microleakage more than half of the depth of cavity wall;

Score 3: Leakage of dye involving axial wall.

The obtained data were tabulated and statistically evaluated using SPSS Statistics for Windows, Version 21.0 (IBM Corp., Armonk, NY) with one-way analysis of variance (ANOVA), and post hoc test, at significance of $p<0.05$.

\section{RESULTS}

Table 1 indicates the highest mean compressive strength for hybrid composite, $250.64 \pm 4.32 \mathrm{MPa}$, followed by $205.12 \pm 1.46$ $\mathrm{MPa}$ in Cention-N and least for GIC, i.e., $167.43 \pm 1.67 \mathrm{MPa}$, which is statistically significant $(p<0.001)$. Table 2 indicates the highest mean flexural strength for hybrid composites (111.78.76 \pm 1.87 $\mathrm{MPa})$ followed by Cention-N (95.67 $\pm 1.24 \mathrm{MPa})$ and least with GIC $(18.43 \pm 1.65 \mathrm{MPa})$, which is statistically significant $(p<0.001)$ Table 3 indicates intergroup comparison of compressive and flexural strength which is highly significant at 0.001 . Table 4 indicates the highest mean microleakage for hybrid composite $(1.27 \pm 0.28)$ and for GIC $(0.35 \pm 0.26)$ and least for Cention-N (0.28 \pm 0.18$)$.

This study indicates highest compressive and flexural strength with hybrid composite, followed by Cention- $\mathrm{N}$ and $\mathrm{GIC}$ restorative materials. There was least microleakage found for Cention- $N$ compared with other restorations.

\section{Discussion}

The primary goal of dental restorative material is to replace functional, esthetic, and biological properties of the healthy
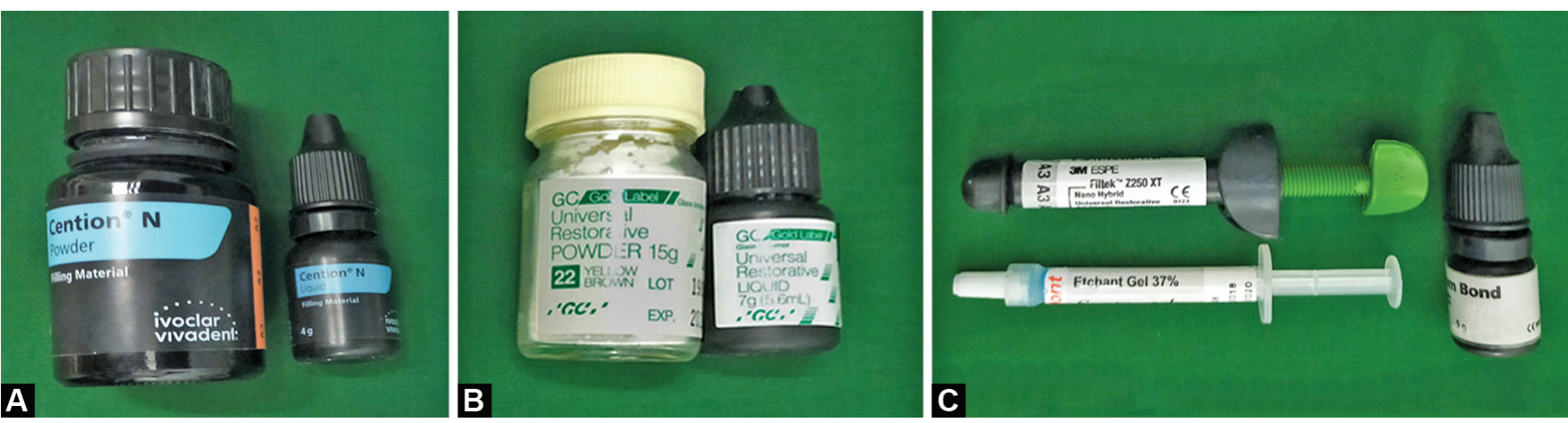

Fig. 1: Materials used: (A) Cention-N; (B) GIC; (C) Hybrid composite 


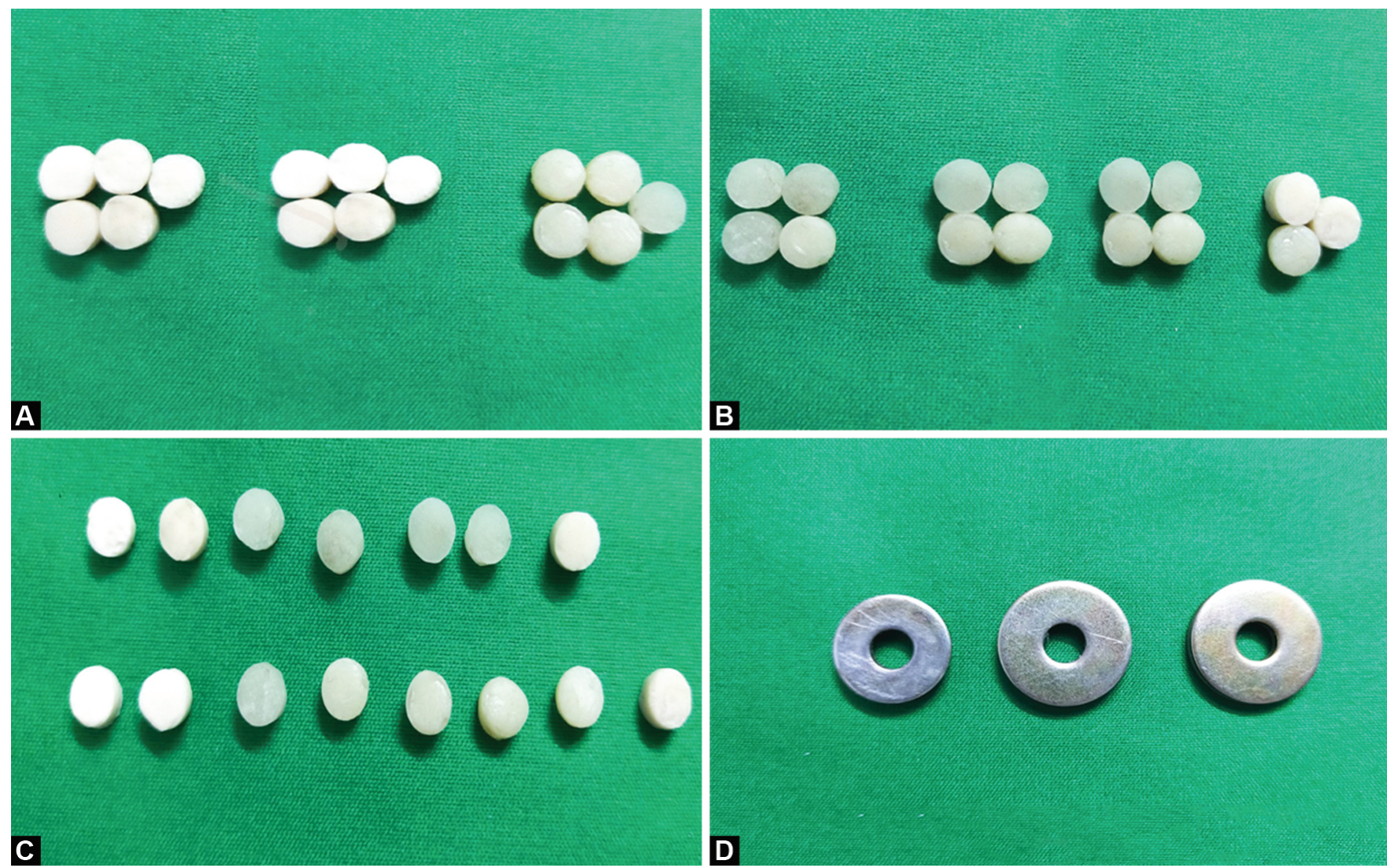

Fig. 2: Restorative material specimen blocks: (A) GIC; (B) Cention-N; (C) Hybrid composite; (D) Metal molds

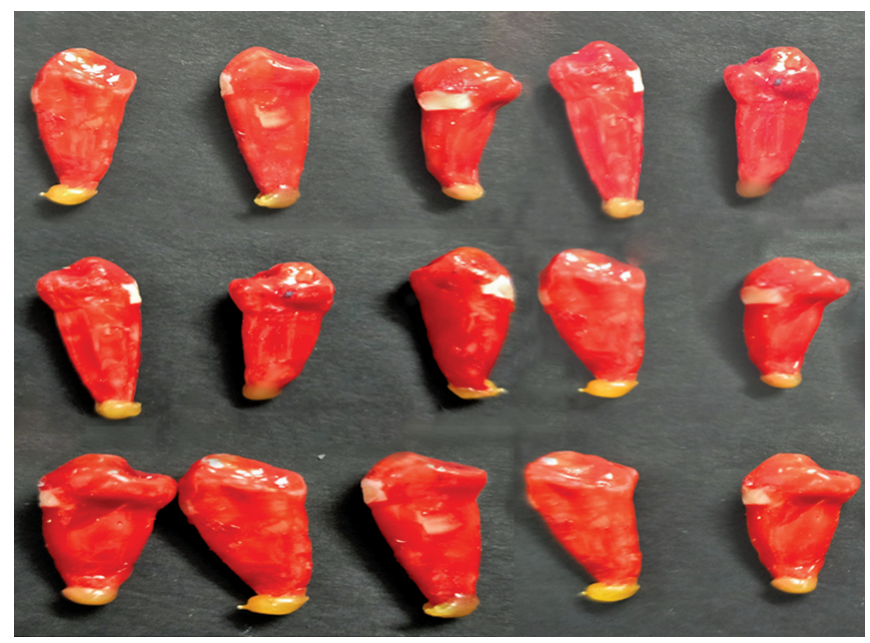

Fig. 3: Premolar teeth specimen with GIC restoration

tooth structure. Stronger restorative materials resist fracture and deformation and provide equal stress distribution, greater stability, and greater clinical success. Compressive and flexural strength have been widely used to evaluate clinical performance of restorative materials. Compressive strength is important for restoration since it provides resistance to compressive and tensile forces produced during mastication. Flexural force is used to evaluate the amount of distortion expected under bending stress. $^{3}$

The present study was done to compare and evaluate the mechanical properties and microleakage of Cention-N with GIC and hybrid composite restorative materials. We found comparatively

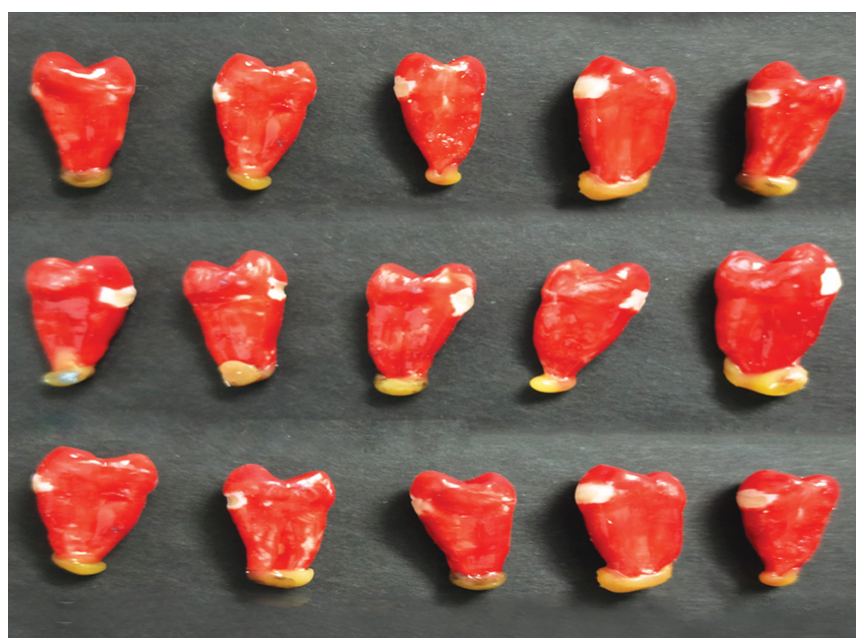

Fig. 4: Premolar teeth specimen with composite restoration

better compressive and flexural strength of Cention-N over GIC but lesser to hybrid composites. Our results are in accordance to findings of Meshram et al., who found mechanical properties of Cention-N was higher than GIC and amalgam but lower than composites. It could be because of micromechanical bonding of resins to tooth structures and resin composites acts as stress breaker. ${ }^{3}$ We observed comparable flexural strength with Cention-N and hybrid composites because of composition of monomer (UDMA) and higher filler content and modulus of elasticity nearer to the tooth structure. Peutzfeldt and Asmussen in their study showed that monomer containing bisphenol A glycol dimethacrylate (BisGMA), triethylene glycol dimethacrylate (TEGDMA), substituted 


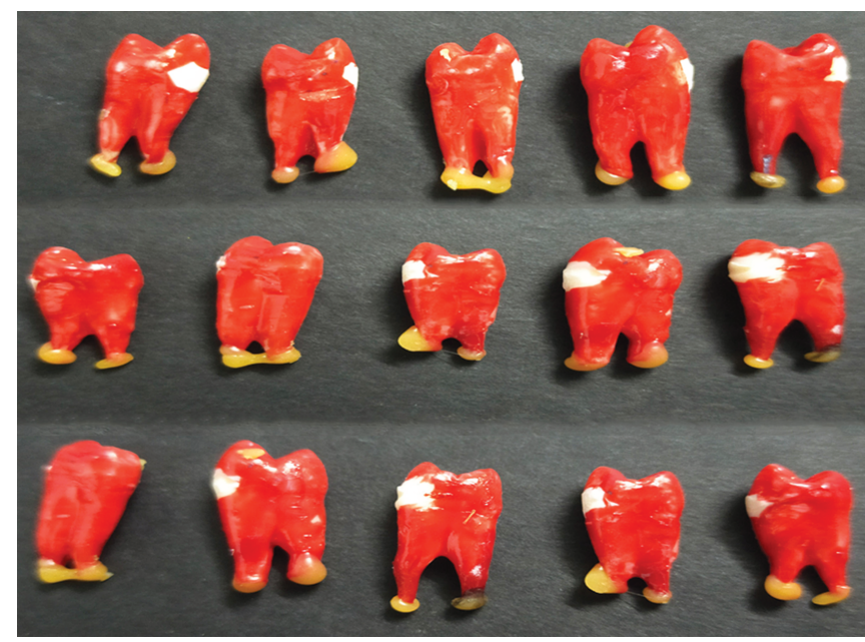

Fig. 5: Premolar teeth specimen with Cention restoration

Table 1: Mean value of compressive strength (MPa)

\begin{tabular}{llll}
\hline Material & $n$ & Mean $\pm S D$ & $p$ \\
\hline Cention-N & 15 & $205.12 \pm 1.46$ & $0.001^{*}$ \\
Nanohybrid composite & 15 & $250.64 \pm 4.32$ & $0.001^{*}$ \\
GIC & 15 & $167.43 \pm 1.67$ & $0.001^{*}$ \\
\hline
\end{tabular}

$p<0.001$; test used: one-way ANOVA, $F$ value: 4247.69 , and post hoc test; $\mathrm{SD}$, standard deviation

*Indicates highly significant

Table 2: Mean value of flexural strength (MPa)

\begin{tabular}{llll}
\hline Material & $n$ & Mean $\pm S D$ & $p$ \\
\hline Cention-N & 15 & $95.67 \pm 1.24$ & $0.001^{*}$ \\
Nanohybrid composite & 15 & $111.78 .76 \pm 1.87$ & $0.001^{*}$ \\
GIC & 15 & $18.43 \pm 1.65$ & $0.001^{*}$ \\
\hline
\end{tabular}

$p<0.001$; test used: one-way ANOVA, $F$ value: 1032.56 , and post hoc test; $\mathrm{SD}$, standard deviation

*Indicates highly significant

Table 3: Inter-group comparison of compressive and flexural strength (MPa)

\begin{tabular}{llcl}
\hline Test & Group comparison & Mean difference & $p$ \\
\hline $\begin{array}{l}\text { Compressive } \\
\text { strength }\end{array}$ & Group I with group II & -45.52 & $<0.001^{*}$ \\
& & & \\
& Group I with group III & 37.69 & $<0.001^{*}$ \\
& Group II with group III & 83.21 & $<0.001^{*}$ \\
Flexural & Group I with group II & -16.11 & $<0.001^{*}$ \\
strength & & & \\
& Group I with group III & 77.24 & $<0.001^{*}$ \\
& Group II with group III & 93.35 & $<0.001^{*}$ \\
\hline
\end{tabular}

*Indicates highly significant

by urethane dimethacrylate (UDMA) has an increased flexural strength. ${ }^{7}$

Kaur et al. found highest compressive strength for Cention-N over GIC type IX from their in vitro study, which is similar to our findings. ${ }^{4}$ Mazumdar et al. observed highest microhardness with Cention compared with amalgam and composite materials. ${ }^{8}$ Kumar and Ajitha showed that there is no significant difference in compressive strength of Cention and high copper amalgam. ${ }^{9}$ Sadananda et al. also found superior compressive and flexural
Table 4: Comparison of mean value of microleakage

\begin{tabular}{llll}
\hline Material & $n$ & Mean $\pm S D$ & $p$ \\
\hline Cention-N & 15 & $0.28 \pm 0.18$ & 0.05 \\
Nanohybrid composite & 15 & $1.27 \pm 0.28$ & \\
GIC & 15 & $0.35 \pm 0.26$ & \\
\hline
\end{tabular}

$p<0.05$; test used: one-way ANOVA; SD, standard deviation

values of Cention over GIC and Zirconomer. ${ }^{10}$ In contrast to our results, Mazumdar et al. observed higher compressive strength with Cention over composite. ${ }^{11}$

Restoration of class $\mathrm{V}$ cavities are always challenging at the dentin interface because gingival marginal portion of dentin has higher organic content, fluid pressure, tubular structure, and lower surface energy. Adequate marginal seal with lower microleakage helps for successful restoration. We used dye material for detection of microleakage since diameter of dye molecule is $0.80 \mathrm{~nm}$ which is lesser than the diameter of dentinal tubule $(1-4 \mu \mathrm{m}) .^{3}$ In the present in vitro study, we have performed thermocycling to simulate the variation in thermal changes in the oral environment.

In the present study, we found lowest microleakage with Cention-N compared with GIC and hybrid composites. Meshram et al. from their in vitro study found that Cention- $\mathrm{N}$ with adhesive has lesser microleakage when compared with without adhesive and flowable composite. They found lesser microleakage at the enamel restoration interface compared with dentin restoration interface. ${ }^{3}$ In contrast to our study, Yazici et al. and Peutzfeldt and Asmussen found lesser microleakage at enamel restoration with flowable composites since they have low viscosity and high flowability. ${ }^{6,7}$ Similar to our findings, Mazumdar et al. found minimal microleakage with Cention-N compared with amalgam and GIC restorations. ${ }^{5}$ George et al. also observed lower microleakage with Cention-N compared with GIC and composite which is in accordance with our findings. ${ }^{12}$ Sahadev et al. observed lower microleakage with Cention-N compared with Zirconomer. ${ }^{13}$ Dodiya et al. found comparatively lower marginal integrity compared with composite. ${ }^{14}$ Hybrid composite has higher microleakage compared with Cention-N since composite demonstrates polymerization shrinkage with volumetric contractions ranging between 2.6 and $4.8 \%$. Cention- $\mathrm{N}$ exhibits a high polymer network density and degree of polymerization over the complete depth of the restoration due to the use of cross-linking methacrylate monomers in combination with a stable, efficient self-cure initiator. It also includes special filler (Isofiller) which acts as a shrinkage stress reliever minimizing the shrinkage force which is responsible for the low volumetric shrinkage leading to least microleakage. ${ }^{12}$ Higher microleakage with GIC could be due to its high viscosity. Iftikhar et al. compared the mechanical properties of four types of restorative materials such as conventional glass ionomer (Fuji IX), ClearFil AP-X, Filtex $\mathrm{Z} 350-\mathrm{XT}$, and Cention- $\mathrm{N}$ and found highest mechanical properties with ClearFil AP-X, but lowest scores with Fuji IX. ${ }^{15}$ Sahu et al. compared microleakage with amalgam, bulk-fill composite, and Cention- $\mathrm{N}$ restorative material for class I cavities and observed promising results with Cention- $\mathrm{N}$ and least microleakage with amalgam compared with composite. ${ }^{16}$

Cention- $\mathrm{N}$ is a subgroup of composite resin class material available in powder and liquid forms. The powder contain alkaline ions like fluoride and calcium which neutralizes the surrounding acidic ions of restoration, whereas liquid contains monomer that 
improves the flowability of the material and adaption to smear layer. ${ }^{3}$ It contains $78.4 \%$ inorganic filler which gives better compressive and flexural strength with lower shrinkage and reduces stress on cavity walls. ${ }^{3,5}$ It has been observed that liquid part of Cention- $\mathrm{N}$ has four different dimethacrylates (urethane dimethacrylate, tricyclodecandimethanol dimethacrylate, aliphatic-UDMA, and polyethylene glycol), and an initiator which helps in the formation of cross-link during polymerization and polymer density which, in turn, improves its mechanical properties. ${ }^{3}$

From the present study, we found that Cention- $\mathrm{N}$ is a good alternative restorative material, which has promising mechanical properties with lesser microleakage. It can be used for various restorative procedures in routine dental practice. Cention- $\mathrm{N}$ is an esthetically acceptable restoration which requires lesser chair-side time for restoration.

The limitation of the present study is that it is in vitro study, since performance and result of the restorative material differ from ex vivo and in vivo oral conditions. In an in vitro study, oral condition simulation of temperature and moisture cannot be maintained, which differs from the restorative material performance. The physical property was tested on a limited number of materials polymerized with one type of unit. Hence, further clinical studies are required on larger sample size.

\section{ConcLusion}

Cention- $\mathrm{N}$ is a newer restorative material having promising properties having lesser microleakage compared with GIC and composite and comparable mechanical properties with composite.

\section{References}

1. Mishra A, Singh G, Singh SK, et al. Comparative evaluation of mechanical properties of Cention $\mathrm{N}$ with conventionally used restorative materials-An in vitro study. Int J Prosthodont Restor Dent 2018;8(4):120-124.

2. Sakaguchi R, Powers J. Craig's Restorative Dental Materials. 13th ed., Philadalphia; 2011.

3. Meshram P, Meshram V, Palve D, et al. Comparative evaluation of microleakage around Class $V$ cavities restored with alkasite restorative material with and without bonding agent and flowable composite resin: An in vitro study. Indian J Dent Res 2019;30(3): 403-407. DOI: 10.4103/ijdr.IJDR_767_17.
4. Kaur M, Mann NS, Jhamb A, et al. A comparative evaluation of compressive strength of Cention $\mathrm{N}$ with glass lonomer cement: An in-vitro study. Int J Appl Dent Sci 2019;5(1):05-09.

5. Mazumdar P, Das A, Das UK. Comparative evaluation of microleakage of three different direct restorative materials (silver amalgam, glass ionomer cement, Cention N), in Class II restorations using stereomicroscope: An in vitro study. Indian J Dent Res 2019;30(2): 277-281. DOI: 10.4103/ijdr.IJDR_481_17.

6. Yazici AR, Baseren M, Dayangac B. The effect of flowable resin composite on microleakage in Class $\mathrm{V}$ cavities. Oper Dent 2003;28(1):42-46.

7. Peutzfeldt A, Asmussen E. Composite restorations: Influence of flowable and self curing resin composite lining on microleakage in vitro. Oper Dent 2002;27(6):569-575.

8. Mazumdar P, Das A, Guha C. Comparative evaluation of hardness of different restorative materials (restorative GIC, Cention N, nanohybrid composite resin and silver amalgam) -an in vitro study. Int J Adv Res 2018;6(3):826-832. DOI: 10.21474/IJAR01/6737.

9. Kumar KS, Ajitha P. Evaluation of compressive strength between Cention $\mathrm{N}$ and high copper amalgam - An in vitro study. Drug Invention Today 2019;12(2):255-257.

10. Sadananda V, Shetty C, Hegde MN, et al. Alkasite restorative material: flexural and compressive strength evaluation. Research Journal of Pharmaceutical, Biological and Chemical Sciences 2018;9(5):2179-2182.

11. Mazumdar P, Das A, Mandal D. Comparative evaluation of bond strength of composite resin \& cention $\mathrm{n}$ to enamel and dentin with and without etching under universal testing machine. University $J$ Dent Scie 2018;4(3):1-6.

12. George P, Bhandary S. A comparative microleakage analysis of a newer restorative material - An ex vivo study. IOSR Journal of Dental and Medical Sciences (IOSR-JDMS) 2018;17(12):56-60.

13. Sahadev CK, Bharath MJ, Sandeep R, et al. An-invitro comparative evaluation of marginal microleakage of cention-n with bulk-fil SDR and zirconomer: a confocal microscopic study. Int J Sci Res 2018;7(7):635-638.

14. Dodiya PV, Parekh V, Gupta MS, et al. Clinical evaluation of Cention-N and nano hybrid composite resin as a restoration of noncarious cervical lesion. J Dent Specialities 2019;7(1):3-5. DOI: 10.18231/j. jds.2019.001.

15. Iftikhar N, Devashish, Srivastava B, et al. A comparative evaluation of mechanical properties of four different restorative materials: an in vitro study. Int J Clin Pediatr Dent 2019;12(1):47-49. DOI: 10.5005/ jp-journals-10005-1592.

16. Sahu S, Ali N, Misuriya A, et al. Comparative evaluation of microleakage in class I cavities restored with amalgam, bulk-fill composite and Cention- $\mathrm{N}-\mathrm{An}$ in vitro confocal laser scanning microscope study. Int J Oral Care Res 2018;6(1):S81-S85. 